\title{
Crear: especular y subvertir
}

\author{
Create: speculate and subvert
}

Recibido: mayo 2019

Aceptado: septiembre 2020

Maritza Granados-Manjarrés ${ }^{1}$

\section{Resumen}

¿Dónde está su lugar de creación? Este artículo establece que, para hacer propuestas innovadoras en el proyecto arquitectónico, los diseñadores deben romper las leyes de lo que saben, ver más allá de lo que se da y subvertir el statu quo.

La propuesta se sitúa en el cruce entre el ejercicio proyectual y los procesos cognitivos detrás de la creatividad, invitando a proyectar desde la ruptura que supone pensar universos aparentemente inconexos.

Para respaldar esa propuesta, proporcionamos un marco teórico para entender la creatividad en arquitectura a la luz de la Teoría de Sistema de Csikszentmihalyi, definimos el tipo de problemas que resolvemos como arquitectos y presentamos la Teoría de Campos de Bourdieu para explicar el funcionamiento del contexto social que avala la incorporación de propuestas arquitectónicas en el universo de lo creado. A partir de esto, proponemos que el uso de estrategias fundamentadas en procesos cognitivos, como el pensamiento divergente o el pensamiento metafórico, pueden promover dichas rupturas y permitirán la aparición de propuestas innovadoras que desestabilizarán esos campos e impulsarán un avance en la disciplina.

\section{Palabras Clave:}

Creatividad; cognición; subversión; proyecto

\begin{abstract}
Where is your place of creation? This article establishes that to make innovative proposals in the architectural project, designers must break the laws of what they know, see beyond what is given, and subvert the statu quo.

The proposal is understood at the junction between the projective exercise and the cognitive processes behind creativity, inviting to project from the rupture that thinking about apparently unconnected universes supposes.

To support that proposal, we provide a theoretical framework to understand creativity in architecture in light of Csikszentmihalyi's System Theory, define the type of problems we solve as architects, and present Bourdieu's Field Theory to explain the functioning of the social context. From there, we propose that the use of strategies based on cognitive processes, such as divergent thinking or metaphorical thinking, can promote these ruptures and will allow the appearance of innovative proposals that will destabilize these fields and will promote progress in the discipline.
\end{abstract}

\section{Keywords:}

Creativity; cognition; subversion; project

\footnotetext{
${ }^{1}$ Nacionalidad: colombiana; adscripción: Profesora Asistente en la Pontificia Universidad Javeriana. Colombia; Doctorado en Arquitectura, Universidad Politécnica de Valencia; e-mail: granados.m@javeriana.edu.co
} 


\section{INTRODUCCIÓN}

En 1976 Bernard Tschumi escribió 'Arquitectura y Transgresión' intentando provocar a los arquitectos alrededor del mundo para que vieran más allá de las reglas de la arquitectura mientras exploraban la relación entre concepto y experiencia en el campo. Cuarenta años después, la transgresión todavía se espera, especialmente cuando la veneración de íconos contemporáneos y la dominación de la imagen ha causado que la arquitectura pierda su habilidad de transgredir y subvertir, al punto que Tschumi nos llama la atención diciendo,

La transgresión es un concepto fundamental, como el límite entre la vida y la muerte. Siempre ha estado ahí y siempre estará. Lo que cambia es su naturaleza (...) siempre habrá nuevos modos de transgresión, a pequeña o gran escala, sociales o filosóficos (Mosley y Sara, 2013:36-37)

Sin embargo, ¿cómo se supone que reconozcamos la relevancia de la transgresión, rompamos con el statu quo, y finalmente podamos subvertir la disciplina para poder avanzar? Proponemos que es desde los estudios de la creatividad desde donde esto debe ser abordado pues en nuestra disciplina crear es siempre un acto de especulación y aunque el especulador fluctúa con la esperanza de beneficiarse, también prevé oportunidades vastas y variadas. Dicho de otro modo, proponemos que pensar el robustecimiento de la arquitectura requiere una mirada profunda y arriesgada cuyo beneficio será el avance de la disciplina, pues solo en la ruptura del establishment puede haber evolución.

Ahora, para alcanzar dicha evolución debemos enfrentar procesos creativos más lúcidos. Por su naturaleza, los problemas en arquitectura y diseño son los escenarios ideales para el estudio de la creatividad, y normalmente se asume que, siendo por definición un campo creativo, ésta es a todas luces considerada. Sin embargo, por la novedad de la epistemología de la arquitectura, en América Latina pocos estudios se han realizado alrededor de los procesos creativos implicados en el ejercicio proyectual arquitectónico por lo que a lo largo de este escrito, vamos a concentrarnos en delimitar cómo los procesos creativos que se dan en la arquitectura y propondremos la subversión como el dispositivo de avance, pues creemos que la ruptura y ese posible adyacente son producto de procesos creativos más consientes.

\section{CREATIVIDAD}

Los estudios sobre creatividad han aumentado en los últimos años debido a sus implicaciones en las artes, la educación, la ciencia, la innovación y la sociedad, y aunque alrededor de la creatividad ha habido una constante reflexión, la investigación sobre ésta es relativamente nueva, sobre todo porque la pregunta sobre la creatividad por dos siglos fue independiente de la institucionalización de la investigación. Esto quiere decir que, 150 años después de que la investigación fuera reconocida y fomentada, emerge el concepto de creatividad, fruto de decenas de debates sobre su definición y separación de conceptos como genio, imaginación $\mathrm{u}$ originalidad de modo que al tratar de definirla empiezan a aparecer particularidades sobre la dificultad de hacerlo de forma resumida y concreta, tomando más bien forma de un intrincado sistema de engranajes cuyo funcionamiento depende de la actividad y precisión de cada una de sus piezas. No obstante, en general sociólogos y antropólogos la van a definir mayormente como un fenómeno sociocultural, y un sinnúmero de teorías van a aparecer para explicar la diversidad de aproximaciones a su definición (Kozbelt et al., 2010).

Ahora, teniendo en cuenta dicha diversidad este artículo propone que, en arquitectura, y en general en las disciplinas proyectuales, la creatividad se entiende como un sistema recursivo que responde a una diversidad de componentes enmarcados en teorías cognitivas, de resolución de problemas y que puede explicarse con el Modelo de Sistema propuesto por Mihaly Csikszentmihalyi (2014).

\section{Modelo de Sistema}

En las observaciones realizadas por Morris Stein en los 50s y los estudios de Dean Simonton en los 90s, quedó comprobado que los eventos sociales, políticos y económicos tenían gran influencia en los índices de producción creativa y que para explicar por qué, cómo y cuándo se producían nuevas ideas o productos y se establecían en una cultura, estos eventos debían ser tenidos en cuenta (Csikszentmihalyi, 2014).

Fruto de esa reflexión es el Modelo de Sistema (Figura 1) que entiende que la creatividad se da 
en la interacción de tres componentes necesarios: el dominio (conocimiento sobre una disciplina), el sujeto creativo y el campo (otros miembros de la disciplina que deciden qué novedades serán incluidas en el dominio). Esto significa que la creatividad ocurre cuando un sujeto creativo puede cambiar un dominio, y este cambio es transmitido a través del tiempo, una vez es aprobado por un grupo que toma decisiones sobre qué puede ser incluido o no dentro del dominio, es decir por el campo, que es su organización social. Para la arquitectura esto querrá decir que un novato o arquitecto en formación recurriendo al acervo teórico-formal de la disciplina producirá novedades que los practicantes ya instalados en esta avalarán y decidirán si deben o no hacer parte del dominio.

Es por esta interacción circular es que se propone como Modelo para entender la creatividad en arquitectura pues a diferencia de otras propuestas que limitan la creatividad a un proceso mental, este modelo la entiende como un proceso tanto social y cultural como psicológico, y está claro que no podemos entender la arquitectura sin su contexto.

En otras palabras, de las perspectivas sobre procesos creativos propuestas en los últimos años, este modelo tiene en cuenta todas las variables que pueden afectar la creatividad, particularmente el contexto social que debe ser reconocido para subvertirlo, pues como se verá, la resistencia solo es posible si se conocen su normas, reglas y limitaciones.

Prueba de las implicaciones del contexto son los proyectos Nueva Babilonia, Instant City y The Walking City que en los años sesenta $\mathrm{y}$ setenta, presentaron nuevos escenarios de crítica política y social; o Archigram y Constant que propusieron estructuras para liberar a la ciudad y disociarla del territorio. A su vez, en 2005 surge el Movimiento Tinyhouse como consecuencia del huracán Katrina y la crisis financiera, proponiendo hogares que no superaran los $9 \mathrm{~m}^{2}\left(96,8 \mathrm{ft}^{2}\right)$.

Conociendo entonces ese contexto social, cultural e histórico y siguiendo el llamado que hace Didier Fiuza Faustino en la XXI Bienal de Venecia para cuestionar el lugar legítimo de la arquitectura como un lugar de acción política y de exploración de los límites formales y mentales ¿cómo transgredimos el estado de las cosas y lo subvertimos para que la disciplina avance?

Figura 1. Modelo de sistema

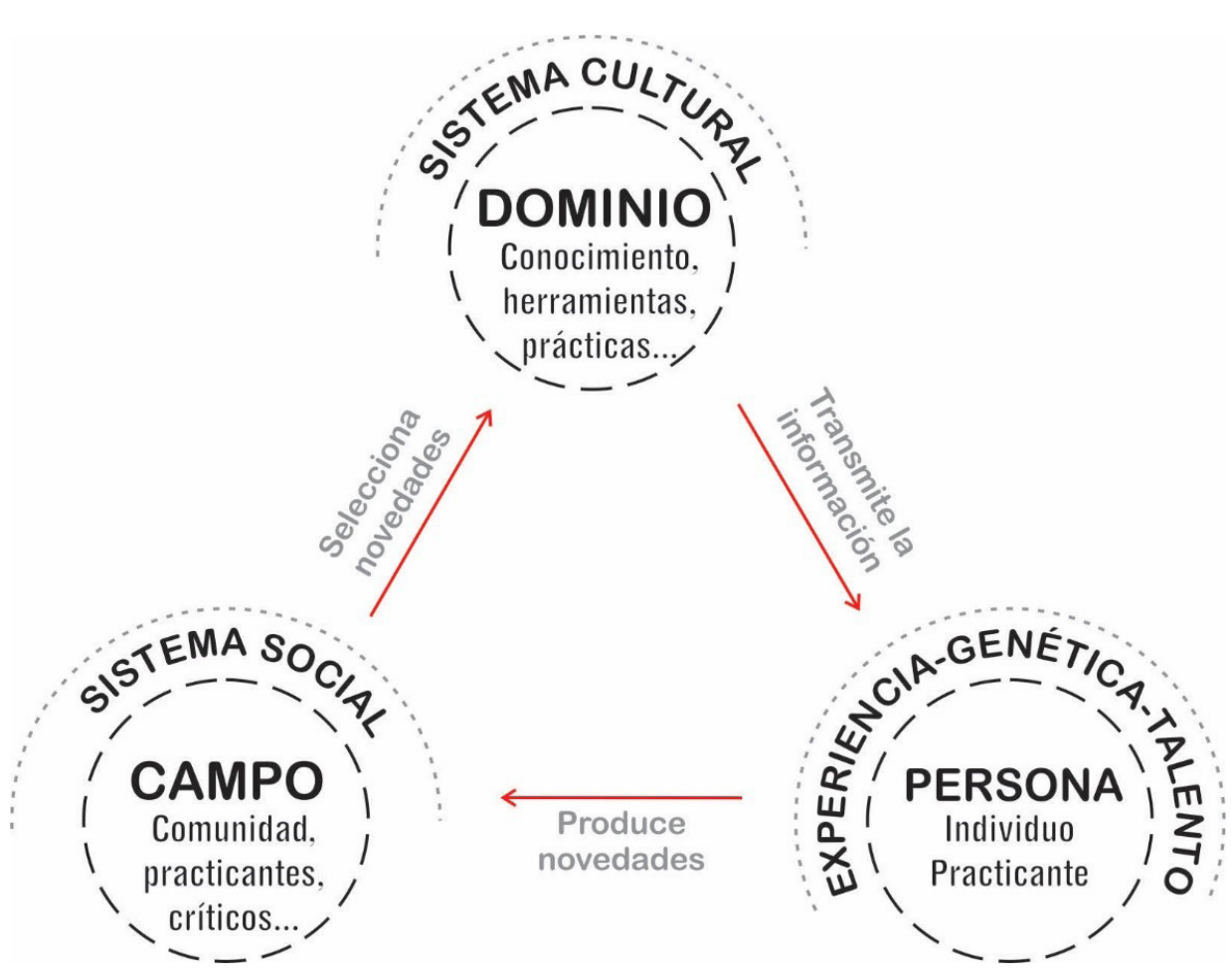

Fuente: Adaptado de: Simonton, Dean (2014), The Wiley Handbook of Genius. Noboken: Wiley-Blackwell, p. 538 


\section{CREATIVIDAD Y SUBVERSIÓN}

Para entender cómo subvertimos el statu quo de la arquitectura hay que empezar por definir qué es lo que resolvemos como arquitectos e identificar los espacios de solución para el cambio. Así que primero vamos a decir que, a diferencia de otras disciplinas donde los problemas son consignas específicas con objetivos específicos, en las disciplinas proyectuales los problemas se definen como 'imprecisos' (ill-defined) y como tal son problemas ambiguos que presentan informaciones opuestas y pueden llevar a diferentes soluciones o situaciones donde ni siquiera es claro si el problema existe (Reitman et al., 1964), definición que a pesar de tener más de 50 años sigue aún vigente.

Además de ello, en las disciplinas proyectuales los problemas deben construirse, un proceso en el cual son definidos, estructurados y sus parámetros son delimitados y re-expresados en tantas formas como sea posible, antes de resolverlos (ReiterPalmon, 2017). Sin embargo, su construcción no es garantía de un resultado creativo.

De acuerdo con Akin y Akin (1996) el diseñador debe romper o violar intencionalmente las restricciones del problema que aborda. Esta pareja, formuló una investigación en la que tomaron el problema de los nueve puntos y lo convirtieron en un problema de arquitectura. El problema original pide unir 9 puntos con 4 líneas rectas sin levantar el lápiz (Figura 2) y para solucionarlo se deben extender las líneas más allá del cuadrado que forman los puntos, que por lo general es a lo que se restringen quienes tratan de resolverlo.

Figura 2. Problema de los 9 puntos resuelto

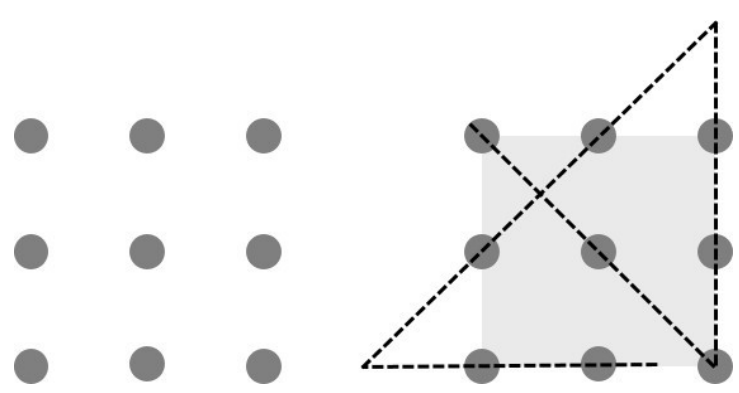

Fuente: Ilustración de la autora
Su versión arquitectónica consistió en rediseñar una fachada existente para una oficina con cinco espacios, en la que participaron 8 sujetos ( 4 novatos y 4 expertos). Su hipótesis fue que a menos que se rompiera el marco de referencia del alzado, no había muchas posibilidades de llegar a un diseño creativo, hipótesis que se comprobó al analizar los resultados del estudio en que concluyeron que la habilidad de los diseñadores de ver más allá de esos marcos hizo posible la aparición de soluciones innovadoras (Figura 3 ). No obstante, aunque pueda deberse a una cuestión de experiencia en un dominio, desde un punto de vista sociológico dicha subversión tiene un funcionamiento, es decir no basta con solo la voluntad de querer promover la indisciplina, hay que saber cómo perturbar lo establecido para poder generar un cambio.

Figura 3. Propuestas de experto y novato
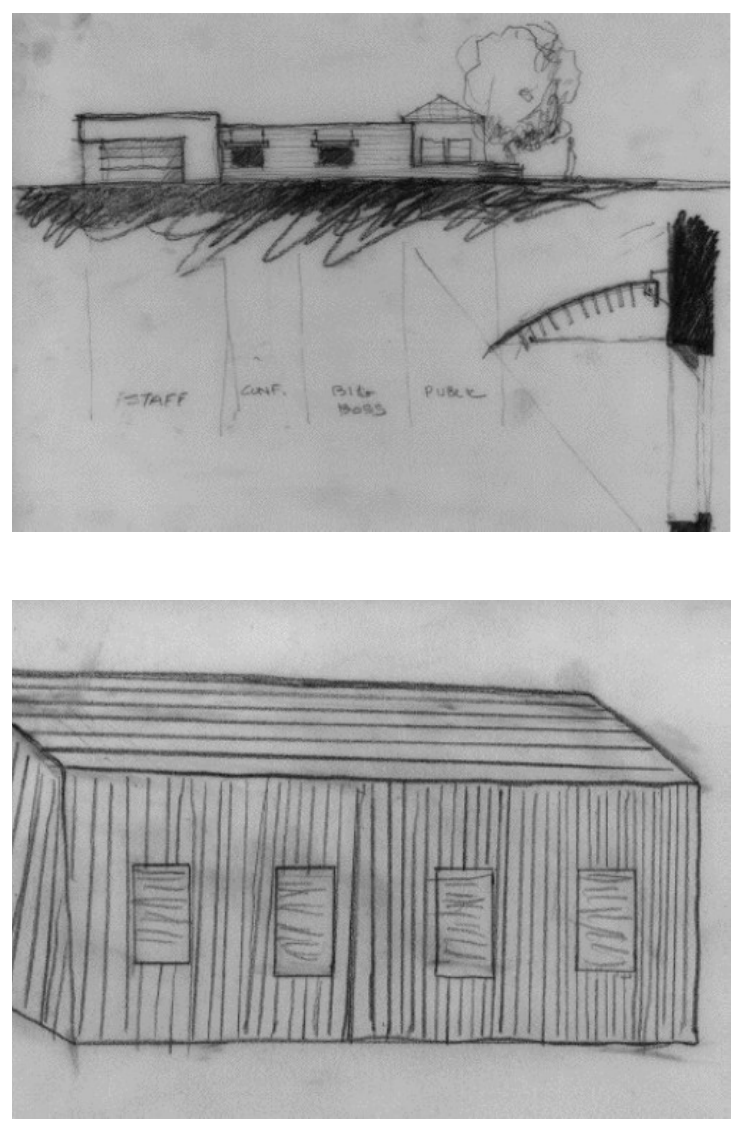

Fuente: Akin, Omer y Akin, Cem (1996). "Frames of Reference in Architectural Design: Analyzing the Hyper-Acclamation (A-h-a-!)", Design Studies. Vol. 17, núm. 4, p. 351. 
Retomando la propuesta de Csikszentmihalyi, sin importar la teoría que se aborde, la mayoría de psicólogos van a estar de acuerdo en que para que algo sea evaluado como creativo debe ser evaluado socialmente y para el Modelo de Sistema esta tarea es llevada a cabo por la organización social del dominio que está formado por profesores, curadores, graduados, críticos y demás practicantes de una disciplina que deciden que productos son considerados creativos o si son suficiente mejora para ser para ser incluidos en el dominio. En muchos sentidos el modelo es análogo al modelo que describe el proceso de evolución que ocurre cuando un individuo produce una variación que es seleccionada por su medio y transmitida a la siguiente (Csikszentmihalyi, 2014).

Por otrolado, la sociedad en símisma representa un elemento fundamental en la creatividad, pues aquella que disfruta de economías estables está en mejores condiciones de ayudar a los procesos creativos en la medida en que una sociedad con mayores riquezas hace que la información esté disponible más rápidamente, se puede permitir la experimentación y especialización y está mejor equipada para implementar nuevas ideas. A su vez es beneficioso que dicha sociedad permita la diversidad cultural pues la sinergia de diferentes ideas es vital en el proceso creativo, y que además permita innovación; dicho de otro modo, las condiciones ideales serán un sistema social que este diferenciado en campos y roles específicos pero se sostenga de lo que Durkheim (2014) definió a finales de 1800 como "solidaridad orgánica". Sin embargo, habrá que preguntarse, ¿cómo se acepta una creación en el dominio? ¿quién tiene la potestad para decidirlo? El Modelo propone que el dominio está protegido por guardianes (gatekeepers en el original) que tienen el derecho y que son colectivamente designados por el campo (Csikszentmihalyi, 2006).

Entonces sugerir cambios, avances y rupturas en una disciplina requerirá en muchos casos desestabilizar el papel de aquellos guardianes que, aunque hayan sido designados por el campo pueden estar obstaculizando, incluso sin saber, los procesos de avance al acomodarse a los privilegios que les da el campo en el que actúan.
Esta coyuntura puede ser explicada a partir de las propuestas de Pierre Bourdieu que en sus estudios socioculturales analiza la sociedad como un conjunto de campos relacionados cuyos actores se enfrentan por los privilegios de cada uno de ellos.

\section{La ruptura}

Aquellos que desafian, obligan a la vieja guardia a montar una defensa de sus privilegios; esa defensa, entonces, se convierte en motivo de subversión. (David Zwarts sobre Bourdieu)

Alejándose del pensamiento sustancialista que propone que las prácticas de los actores son producto directo de su posición social, Bourdieu propone que en la sociedad existen estructuras que afectan las prácticas de sus actores y esa afección es mediada por la subjetividad deestos, es decir plantea lo social como una estructura multidimensional, no como un espacio determinado únicamente por sus relaciones económicas, y para explicarlo plantea las categorías de campo, capital y habitus.

De acuerdo con Bourdieu un campo se determina por un capital (económico, social, simbólico, cultural) que es común a sus actores y por la lucha por apropiarlo, de modo que el campo se constituye por un sistema de posiciones sociales tanto como de relaciones de fuerza entre tales posiciones. Esto quiere decir que en un campo las relaciones objetivas entre diferentes posiciones le imponen a sus ocupantes "una determinada situación en la estructura de la distribución de las clases de poder o capital cuya posesión determina acceso a específicos beneficios inherentes a cada campo, así como una relación de acuerdo a otras posiciones" (Chihu Amparán, 1998:182-183). En estos campos donde un individuo se posiciona de acuerdo con su capital se crea un espacio de tensiones con otros de su tipo para retener o cambiar la distribución de poder producto del capital inherente al campo que se disputa. De hecho, su estructura es un momento histórico en el que la distribución de un capital específico se ha hecho, y es consecuencia de las muchas batallas peleadas por los agentes involucrados

\footnotetext{
2 Se definen como sociedades donde los individuos interpretan roles distintos y su unidad se basa en sus diferencias y no sus similitudes, ejemplo de ello son las sociedades industriales donde los roles están necesariamente interconectados y su desempeño en la multiplicidad es vital para que la sociedad funcione.
} 
en el campo; esto significa que la estructura es también las relaciones entre actores e instituciones involucradas en el juego (Gutiérrez, 2012).

Por su parte, el habitus será el conocimiento de las disposiciones de los actores de un campo que se relacionan con las reglas que lo legitiman y que son sistemas que dan cuenta de los rasgos de una posición social que se manifiestan en sus prácticas, interacciones y formas de comportarse que establecen diferencias con otros actores y sus prácticas sociales.

En otras palabras, el campo es un dominio que responde a reglas y contiene las instituciones que dan sentido a las relaciones entre sus actores y su posición relativa definida por la estructura y volumen de su capital. Al reconocer la arquitectura como un campo, puede también reconocerse la autonomía que le ha permitido la generación de élites responsables de legitimar cada acción dentro de dicho campo, y esta autonomía permite la emergencia de un capital que es capital de reconocimiento. Esto significa que hay una lucha "por imponer una definición de reconocimiento legítimo, en el cual la victoria conduce a un control monopolizado de la definición de las formas de legitimidad que prevalecen en el campo" (Hilgers y Mangez, 2015:6).

Piense por un momento en esto. Cada dos o tres años, cerca de 50 festivales, bienales y trienales muestran lo mejor de lo que pasa en el mundo de la arquitectura. Desde 1979 el Premio Pritzker, reconoce a un arquitecto vivo cuya obra demuestre la combinación de talento, visión y compromiso con gran significado para la humanidad y el entorno construido; y además define quién es quién en el mundo de la arquitectura.

En 1991 Robert Venturi fue galardonado en solitario a pesar de haber trabajado muy de cerca con Denise Scott Brown quien no fue reconocida con la excusa de que los premios no se hacían colectivamente, situación que dejó de ser importante al galardonar a Herzog y de Meuron porque no podrían haber hablado de uno sin el otro, pero ¿quién toma esa decisión? Usualmente un grupo de entre 5 y 9 miembros que son en su mayoría hombres, y que por casi 10 años no incluyó a ninguna mujer (Heynen, 2012).

Este es un ejemplo de cómo la propuesta de Bourdieu explica parte del funcionamiento del ejercicio de la arquitectura al afirmar que los actores en el campo están normalmente bien establecidos en él y están interesados en mantener el orden instaurado, o al menos modificarlo de modos que fortalezcan su dominación. Los nuevos podrán no tener oportunidad de alterar ese campo, sin embargo, la subversión moviliza grupos, la subversión puede activar estructuras que promuevan otras lógicas y apuestas.

Si entendemos la arquitectura como un modo único de provocación, el ejercicio proyectual debería promover variedades de actos subversivos que desestabilicen los campos en los que se inscriben los diversos actores a través de hibridaciones alejadas de los lugares comunes del diseño, poéticas críticas y progresivas y especulaciones contestatarias de la realidad oficial normalizada.

Esa desestabilización podrá tomar muchas formas en el ejercicio de proyecto, pero vamos a proponer que proyectos afianzados en el reconocimiento del cruce de sus variables permitirán la emergencia de fenómenos, experiencias estéticas, vértigos y utopías, sugiriendo interacciones más agudas que amplían lo visible y develan aquello que el sistema no quiere que sea visto.

Para ello vamos a proponer la integración del pensamiento divergente y el pensamiento metafórico en el ejercicio proyectual pues como se verá, han sido probados como herramientas que promueven nuevas perspectivas, conexiones, transgresiones y cambios de pensamiento.

\section{PENSAMIENTO DIVERGENTE}

El pensamiento divergente es entendido como el pensamiento donde se generan diversas soluciones a un problema y según Scott (1999) requiere diferentes alternativas en relación con un solo estímulo, lo que permite la aparición de muchas respuestas, y múltiples alternativas que pueden satisfacer una serie de criterios.

En términos de la arquitectura, Doaa Hassan (2016) propone un estudio que buscaba comparar técnicas representativas del pensamiento divergente basadas en las propuestas de brainstorming de Osborn, y las propuestas de pensamiento lateral desarrolladas por De Bono.

Una de las técnicas propuesta por Bob Eberle y conocida como SCAMPER (Figura 4) es una guía que promueve la aparición de nuevas perspectivas o soluciones a una situación determinada. Cada una de las letras representa una palabra o frase como guía para la formulación de preguntas que, en su resolución, conducen el pensamiento creativo a nuevas áreas o nuevos territorios 
(Gaubinger, Rabl, Swan, y Werani, 2015). La otra técnica, es conocida como Flor de Loto (Figura 5) y es la representación visual de las ideas, como un mapa mental, en el que el diseñador comienza con su problema principal en el centro sobre el que desarrollan 8 sub-problemas, y alrededor de estos se desarrollan 8 ideas más.

En cuanto al pensamiento lateral, en el que las técnicas divergentes son utilizadas para forzar a nuestros cerebros a rechazar patrones preconstruidos a través de procesos aparentemente ilógicos, Hassan escoge la provocación de entre las siete técnicas conocidas. Para ella, esta técnica es la que más promueve que el cerebro funcione en contra su naturaleza, al obligar al diseñador a hacer afirmaciones ilógicas en las que algo que se creía cierto de una situación, no lo es.

Hassan propone que las técnicas como SCAMPER y la Flor de Loto son estructurales, mientras que las de pensamiento lateral son procedimentales y se pregunta cuál lleva a un producto más creativo por lo que propone un estudio que desarrolló en la Universidad de Ain Shams en Egipto durante seis semanas en la cátedra de Teoría de la Arquitectura 2.

Los estudiantes fueron divididos en dos grupos, a cada uno le correspondió una técnica (Flor de
Loto o Provocación), y la consigna consistía en hacer dos proyectos utilizando la técnica que le correspondiera. Hassan llega a la conclusión de que los procesos de diseño arquitectónico deben ser liberados de técnicas tradicionales que sigan los modelos de Análisis/Síntesis y deben adoptar modelos de Conjetura/Análisis que optimizan el concepto de creatividad y que sugieren el uso de técnicas de pensamiento divergente como las comparadas en el estudio realizado. Por otro lado, concluye que las técnicas procedimentales son más útiles en el caso de buscar ideas innovadoras en poco tiempo, mientras que las estructurales son beneficiosas cuando se necesiten muchas ideas, sin embargo, las primeras pueden necesitar mucha más práctica para ser aplicadas debido a su naturaleza conceptual.

Estas técnicas son algunas de las posibilidades para promover el pensamiento divergente y en este caso queda claro que pueden ser pensadas en el ejercicio de diseño, no obstante, podrían también ser utilizadas técnicas de sinéctica, mapas mentales, los 6 sombreros para pensar y hasta el sleepwriting de Dalí. Lo importante será siempre promover la aparición de novedades que en su elaboración y construcción puedan sugerir cambios evolutivos para la disciplina.

\section{Figuras 4 y 5. Scamper y Flor de Loto}
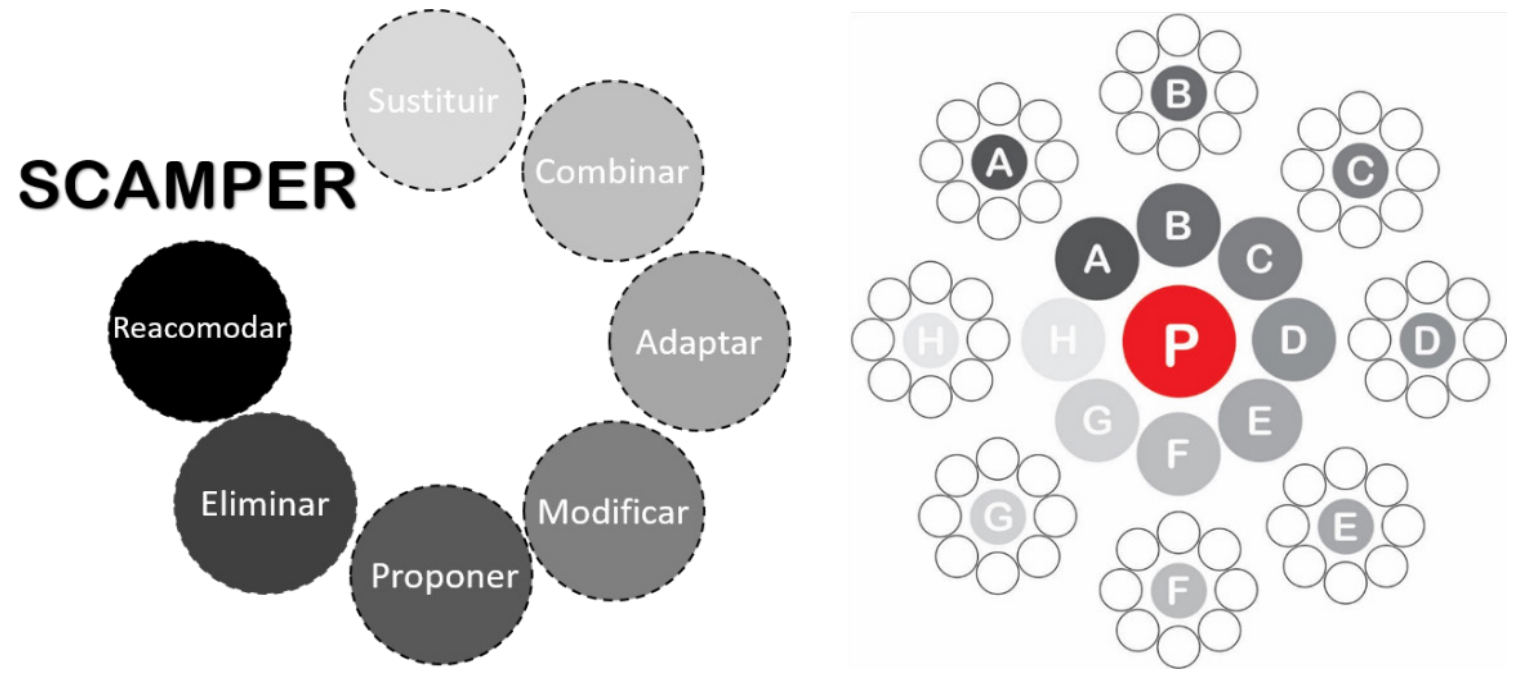

Fuente: Ilustración de la autora 


\section{PENSAMIENTO METAFÓRICO}

Por su parte, para la psicología de la creatividad, el uso de la metáfora está fundamentalmente arraigado en los procesos de pensamiento creativo al afirmar que la asociación entre universos conceptuales independientes es la fuente principal de la creatividad, y que entre más distantes sean dichos conceptos, más productiva será la metáfora en términos de generación de percepción creativa (Sánchez-Ruíz, Romo S., y Jiménez J., 2013).

El arquitecto Hernán Casakin (2007) afirma que las metáforas estructuran el sistema cognitivo, organizando el pensamiento y permitiendo la aparición de nuevas formas de pensar al permitir pensar una situación en términos de otra, constituyendo una yuxtaposición entre lo familiar y lo inusual, actividad de mucha ayuda a la hora de encarar problemas imprecisos.

Es por esto que Casakin, buscando evaluar la creatividad en el proceso de diseño y su comportamiento cuando se utilizaba la metáfora, invitó a 65 estudiantes de primer año a proponer una serie de edificaciones de uso mixto en una zona deteriorada de Tel Aviv.

Después del trabajo proyectual, los estudiantes contestaron una encuesta en la que evaluaban sus proyectos en términos de su fluidez, innovación, funcionalidad, estética, valor, productividad, relación con el contexto físico etc., a la vez que evaluaban el rol de las metáforas en la organización del pensamiento, la búsqueda de relaciones inusuales entre dominios remotos y el problema de diseño, y el análisis del problema desde puntos de vista diferente. Casakin concluye que las metáforas deben ser vistas como una estrategia cognitiva, y los resultados arrojaron que la innovación fue el factor dominante en la creatividad.

Como continuación de este estudio, Hey, Linsey, Agogino y Wood (2008), discuten la relación entre metáfora y analogía en los procesos de diseño, apoyándose en entrevistas y experimentos con estudiantes, para llegar a la conclusión de que ambas son utilizadas normalmente, con la diferencia que la metáfora se emplea en las primeras etapas proyectuales cuando el problema está siendo enmarcado, y la analogía en la generación de conceptos. Además, concluyen que los profesionales las usan frecuentemente, pero los estudiantes deben ser enseñados a emplearlas efectivamente, pues está claro que ambas traen enormes beneficios al ejercicio del diseño, pues el proceso de comparación fomenta la interpretación de problemas de formas novedosas y la aparición de propuestas inusitadas.

Prueba de la fertilidad de esa vinculación entre universos distantes y la ruptura que propician puede rastrearse a lo largo de una variedad de propuestas proyectuales: las arquitecturas líquidas de principios de siglo XXI de Marcos Novak o Karl S. Chu, el diseño morfogenético del Instituto de Diseño y Construcción Computacional de Achim Menges, los cruces entre arte e ingeniería de Anish Kapoory Cecile Balmond o la fractalidad de Serge Salat y Michael Hansmeyer.

Al igual que con el Pensamiento Divergente, al margen de la técnica que se use para promover las metáforas durante los ejercicios de resolución de problemas, lo importante será posibilitar su aparición para nuevamente producir relaciones que en su robustecimiento permitan el avance de la disciplina.

\section{CONCLUSIONES}

Vamos a resumir el camino que acabamos de tomar y la propuesta que surge de hacerlo. Dijimos que la creatividad en arquitectura se enmarca en la triada propuesta por Csikszentmihalyi en tanto es un producto social, histórico y cultural que depende de su contexto y de la validación de sus agentes para poder incluirse en el acervo formal del universo construido. A continuación, llamamos la atención sobre la imprecisión de los problemas que enfrentamos como arquitectos e ilustramos cómo esos problemas demandan una ruptura de sus marcos de referencia para impulsar propuestas innovadoras, ruptura que es posible por la naturaleza misma de los problemas que para ser solucionados demandan una construcción previa.

No obstante, aparecen acá dos cuestiones, por un lado, la posibilidad de acceder a dicho acervo, y por el otro las estrategias cognitivo-formales para lograrlo.

Una vez instalados en la triada de sistema como creadores debemos enfrentar a aquellos que salvaguardan el acceso a un dominio. Tomando la propuesta de Bourdieu sobre la Teoría de Campos, es posible que estos agentes opongan resistencia al estar instalados en un campo donde quieren seguir ejerciendo su dominio y seguir disfrutando de sus privilegios, por lo que las rupturas no deberán ser solo intenciones sino verdaderos cambios que puedan llegar a desestabilizar el establishment. 
Para lograrlo proponemos utilizar el pensamiento divergente y el pensamiento metafórico para la construcción y resolución de problemas en tanto posibilitan la aparición de propuestas disruptivas, innovadoras e inesperadas.

Es importante decir que consideramos que en las prácticas académicas es donde las rupturas se hacen más necesarias para promover revoluciones de pensamiento principalmente por dos cuestiones: por un lado porque tal y como menciona Teymur (2011) la cantidad de libros sobre educación en arquitectura es notablemente menor al número de libros publicados sobre cualquier arquitecto significativo y en la única revista académica dedicada a la enseñanza de la arquitectura, la mayoría de textos no son sobre educación, lo que deja en evidencia la necesidad (e invitación) de contribuir a la constitución de una epistemología y didácticas que nos sean más propias. Por el otro, porque será de la academia de donde surjan esas mentes que puedan desequilibrar el sistema y permitir su avance.

Mikhail Bakhtin define el carnaval como la relajación de las normas regulares en una sociedad, es decir el reconocimiento de unas reglas que después son negadas, lo que nos lleva a pensar ¿qué sería un carnaval en nuestro campo?

Quisiéramos finalizar este escrito con esa pregunta. Queremos proponer que empujar esos límites no es solamente crucial para el avance de la profesión y la academia, sino que dicho carnaval será potenciado si prestamos atención al funcionamiento de los procesos de creación en la medida en que el sujeto creativo se vuelve más consciente de su producción. De hecho, creemos que proponer la interconexión de diversos universos y disciplinas es la clave para subvertir la Arquitectura y prepararla para híbridos posibles. Esta es una invitación a subvertir el statu quo, a cambiar lo que se le ha dado y especular. Lo invitamos a proponer un carnaval. C

\section{REFERENCIAS BIBLIOGRÁFICAS}

Akin, O., \& Akin, C. (1996). "Frames of reference in architectural design: analyzing the hyper-acclamation (aha!)". Design Studies, Vol.17, núm. 4, pp. 341-361.

Casakin, H. P. (2007). "Metaphors in Design Problem Solving: Implications for Creativity". International Journal of Design, Vol. 1, núm. 2, 21-33.
Chihu Amparán, A. (1998), "La Teoria de los campos". Polis, pp. 179-198.

Csikszentmihalyi, M. (2006), "A Systems Perspective on Creativity”, en J. Henry (Ed.), Creative management and development. London, Sage Publishing, pp. 3-17.

Csikszentmihalyi, M. (2014), "Creativity and Genius". En Csikszentmihalyi, M. The Systems Model of Creativity: The Collected Works of Mihaly Csikszentmihalyi. London, Sage Publishing, pp. 99-125.

Gaubinger, K., Rabl, M., Swan, S., y Werani, T. (2015). "Creativity Techniques", en Innovation and Product Management. New York, Springer, pp. 115-131.

Guitiérrez, A. (2012). Las prácticas sociales. Villa María, Eduvim.

Hassan, D. (2016). "Divergent thinking techniques discrepancy and functional creativity: Comparative study of structural and procedural techniques in architectural design". Ain Shams Engineering Journal, pp. 1-15. doi:http://doi.org/10.1016/j. asej.2016.10.002

Hey, J., Linsey, J., Agogino, A., y Wood, K. (2008). "Analogies and metaphors in creative design". International Journal of Engineering Education, Vol. 24, núm. 2, pp. 283-294.

Heynen, H. (2012). "Genius, gender and architecture". Architectural Theory Review, Vol. 17, núm. 2-3, pp. 331-345.

Hilgers, M., y Mangez, E. (2015). "Introduction to Pierre Bourdieu's theory of social fields", en Hilgers, M. y Mangez, E., Bourdieu's Theory of Social Fields. New York, Routledge, pp.1-36

Kozbelt, A., Beghetto, R., y Runco, M. (2010), "Theories of Creativity", en R. Kaufman, James; Sternberg (Ed.), The Cambridge Handbook of Creativity. Cambridge, Cambridge University Press, pp. 20-47.

Mosley, J., \& Sara, R. (2013). "Architecture and transgression: an interview with Bernard Tschumi”. Architectural Design, Vol. 30, núm. 1, pp. 32-37.

Reiter-Palmon, R. (2017). "The Role of Problem Construction in Creative Production". Journal of Creative Behavior, Vol. 51, núm. 4, pp. 323-326. http://doi.org/10.1002/jocb.202

Reitman, W., Shelly, M., \& Bryan, G. (1964), "Heuristic decision procedures, open 
constraints, and the structure of ill-defined problems", en Human judgments and optimality. Londres, John Wiley and Sons, pp. 282-315.

Sánchez-Ruíz, M. J., Romo S., M., \& Jiménez J., J. (2013). "The role of metaphorical thinking in the creativity of scientific discourse". Creativity Research Journal, Vol. 25, núm. 4, pp. 361-368.

Scott, T. E. (1999). "Knowledge”. En Runco, M. y Pritzker, S. Encyclopedia of creativity. Volume 2, San Diego, Academic Press, pp. 119-129.

Teymur, N. (2011). "Aprender de la educación en arquitectura”. Dearq, pp. 8-17. 